\title{
État de la recherche sur le lait de chèvre en Grèce
}

\author{
G Kalantzopoulos \\ Dairy Department, Agricultural University of Athens, 86 lera Odos, Botanikos 11815, \\ Athènes 301, Grèce
}

\begin{abstract}
Résumé - La Grèce est le premier pays de la Communauté européenne pour la production de lait de chèvre (460 000 t/an) avec un cheptel caprin de 5904000 animaux, des races indigènes (85\%). La recherche dans ce domaine est faite par les stations du ministère de l'Agriculture, I'Institut du lait et les facultés relatives des Universités. Elle est orientée au point de vue zootechnique vers l'amélioration génétique des races locales par sélection et croisement. L'étude du lait est également très importante : sa composition moyenne est la suivante : matière grasse de 4,4-5,8\%, protéines 3,4$3,7 \%$, caséine $2,7-2,8 \%$, lactose $4,4-5,0 \%$. En ce qui concerne la valorisation du lait, des études sont faites sur la production du yoghourt pur chèvre, la production de Feta en mélange avec le lait de brebis et sur la production des nouveaux types de fromages.
\end{abstract}

lait de chèvre / recherche / composition / fromage / Grèce

Summary - A review of current research on goat's milk in Greece. Greece is the main producer of goat's milk in the European Community, with an annual output of 460000 ton/yr and a livestock of 5904000 goats (85\% native). Research in the field is carried out by Agricultural Ministry stations, the Milk Institute and the relevant university faculties. The zootechnical aspect is focussed on genetic improvement via selection and cross-breeding of native goat breeds. The study of milk composition is also very important; its average composition is as follows: fat $4.4-5 \%$, total protein $3.4-3.7 \%$, casein 2.7-2.8\% and lactose 4.4-5.0\%, Concerning the transformation of goat's milk, studies are currently in progress on the production of yoghurt from pure goat's milk, Feta cheese composed of a mixture of sheep's milk and goat's milk, and also on the production of new types of cheeses.

goat's milk / research / composition / cheese / Greece

\section{INTRODUCTION}

Historiquement, socialement et économiquement, la chèvre et la brebis jouent depuis l'Antiquité un rôle majeur dans les économies des régions montagneuses et sèches de la Grèce. Du printemps à l'automne les animaux transhumants se pro- curent la majeure partie de leurs besoins alimentaires sur pâturage, mais pendant le reste de l'année, ils sont alimentés avec des concentrés, des céréales, des tourteaux de coton, etc.

La population caprine répertoriée selon les données statistiques du service national de la statistique (1990) est présentée 
dans le tableau I. Le cheptel caprin total est par ailleurs de 5904494 têtes.

En Grèce, comme dans la plupart des pays méditerranéens, il existe une race locale. Des efforts de sélection ont été réalisés ces dernières années pour les stabiliser. Des Saanen et des Alpines ont été importées en nombre limité pour réaliser des croisements avec les populations locales. L'élevage caprin est toujours à finalité mixte : lait et viande. La race locale se caractérise par une tête triangulaire et sans cornes, du type Capra prisca et une bonne conformation. Sa production laitière est moyenne, environ $70 \mathrm{~kg}$ dans le système pastoral mais elle peut atteindre 200 $\mathrm{kg}$ avec une moyenne de $100 \mathrm{~kg}$ en conditions améliorées. Les systèmes d'élevage varient entre l'extensif avec des troupeaux mixtes brebis-chèvres et l'intensif, avec toutes les solutions intermédiaires adaptées aux différents milieux.

La production laitière globale atteint 460000 tan pour une durée de lactation de 6 mois. La Grèce est le premier producteur de la Communauté européenne. La part de chèvre représente $27 \%$ de la production totale du lait de la Grèce.

Une partie notable du lait de chèvre produit est consommée localement, soit en autoconsommation soit commercialisée sous la forme de fromages, yoghourt et beurre fondu sur les marchés de proximité.

La majeure partie du lait est utilisée à la production du fromage Feta, en mélange avec le lait de brebis selon une tradition agricole très ancienne de la Grèce. Les produits frais uniquement à partir de lait de chèvre sont rares à l'échelle industrielle mais fréquents à l'échelle artisanale.

Cela s'explique par 2 raisons : les troupeaux sont mixtes, brebis-chèvres, avec une productivité moyenne; et le lait de chèvre des races locales est riche et a une composition proche de celle du lait de brebis.

\section{L'ORGANISATION DE LA RECHERCHE}

\section{Élevage caprin}

Le ministère de l'Agriculture dispose de 10 stations de recherche d'élevage ovin et caprin. Les principaux thèmes d'étude concernent :

- la maîtrise de la reproduction : synchronisation, insémination artificielle, transfert d'embryon etc... ;

- amélioration génétique des races locales ;

- utilisation de paturages arbustifs.

Les facultés zootechniques de l'Université agronomique d'Athènes et de l'Université de Thessalonique travaillent sur ces mêmes sujets, avec notamment l'identification génétique des qualités de production. Dans ce cadre, le professeur Rogdakis

Tableau I. Distribution de la population caprine traitée.

Distribution of goat populations.

\begin{tabular}{lrrrr}
\hline Mode d'élevage & En plaine & Semi-montagne & Montagne & Total \\
\hline Domestiquées & 624014 & 1140454 & 1741728 & 3506196 \\
Nomades & 52047 & 107200 & 52452 & 211699 \\
Total & 676061 & 1247654 & 1794180 & 3717895 \\
\hline
\end{tabular}


étudie une race locale de l'île Skopelos, présentant une rusticité bien adaptée aux conditions d'une île sèche et aride et ayant un rendement laitier de $170-250 \mathrm{~kg}$ par lactation.

\section{Lait}

La consommation directe de lait en nature est mal connue ; $30 \%$ environ seraient autoconsommés. Cette proportion est certainement valable pour les régions montagneuses, dont l'économie repose sur un système fermé d'autoapprovisionnement.

L'utilisation de la chèvre comme fournisseur de lait et de viande, permettrait de lutter contre la malnutrition en plaçant chez chaque petit éleveur 2-4 chèvres.
L'Institut du lait appartenant au ministère de l'Agriculture étudie la composition du lait produit dans les différentes régions par les différentes populations et races et l'optimisation de la technologie de fabrication des fromages de lait de brebis et de chèvre.

Les laboratoires de technologies laitières des Universités d'agriculture d'Athènes et de Thessalonique travaillent sur ces mêmes sujets et également sur une meilleure valorisation de ces laits, sans oublier l'étude des fromages traditionnels. Des recherches sont également réalisées sur la transformation du lait de chèvre en yoghourt.

Les tableaux II, III et IV présentent la composition du lait de chèvre de Grèce. Nous pouvons constater que la richesse du lait des races locales est très élevée et

Tableau II. La composition du lait de chèvre de races locales des régions Epirus (A) et Argolis (B) selon Anifantakis et al (1993).

Goat's milk composition in local breeds from the regions of Epirus (A) and Argolis (B) (from Anifantakis et al, 1993).

\begin{tabular}{llllll}
\hline Mois $\quad \mathrm{N}$ & $\mathrm{kg} \quad$ Matgrasse Protéine Lactose $P$ cong $\left({ }^{\circ} \mathrm{C}\right) \quad \mathrm{pH}$
\end{tabular}

A. (Nord-ouest de la Grèce)

$\begin{array}{lrrrrrrr}\text { Janvier } & 3 & 12,2 & 5,56 & 3,79 & 4,58 & -0,545 & - \\ \text { Février } & 29 & 29,2 & 5,26 & 3,64 & 4,50 & -0,547 & 6,69 \\ \text { Mars } & 9 & 60,1 & 4,63 & 3,46 & 4,36 & -0,531 & 6,68 \\ \text { Avril } & 42 & 31,3 & 5,33 & 3,63 & 4,48 & -0,542 & 6,62 \\ \text { Mai } & 45 & 52,1 & 5,13 & 3,67 & 4,51 & -0,554 & 6,53 \\ \text { Juin } & 51 & 45,6 & 4,84 & 3,56 & 4,36 & -0,546 & 6,49 \\ \text { Juillet } & 50 & 37,4 & 4,79 & 3,48 & 4,28 & -0,544 & 6,58 \\ \text { Moyenne } & - & 40,7 & 5,00 & 3,58 & 4,41 & -0,546 & 6,57\end{array}$

B. (Grèce du Sud)

$\begin{array}{lrllllll}\text { Janvier } & 95 & 80,1 & 6,44 & 4,07 & 4,65 & -0,551 & - \\ \text { Février } & 88 & 78,7 & 6,55 & 4,03 & 4,57 & -0,552 & - \\ \text { Mars } & 100 & 74,5 & 5,80 & 4,05 & 4,58 & -0,562 & 6,55 \\ \text { Avril } & 96 & 67,3 & 5,77 & 3,97 & 4,52 & -0,552 & 6,56 \\ \text { Mai } & 96 & 82,6 & 5,30 & 3,91 & 4,41 & -0,562 & 6,38 \\ \text { Juin } & 50 & 60,6 & 5,46 & 3,83 & 4,15 & -0,559 & 6,41 \\ \text { Moyenne } & - & 75,1 & 5,92 & 3,99 & 4,51 & -0,557 & 6,48\end{array}$


Tableau III. Composition du lait de chèvre de races locales de différentes régions de Grèce selon les données non publiées, pour les régions Thessalia et loannina (Anifantakis et al; Veinoglou et al, 1982).

Goat's milk composition in local breeds in certain regions of Greece (Thessaly, Macedonia and loannina ; Anifantakis et al ; Veinoglou et al, 1982).

\begin{tabular}{|c|c|c|c|c|}
\hline & \multicolumn{4}{|c|}{ Régions de Grèce } \\
\hline & Ioannina & Thessalia & Macédoine & Attika \\
\hline Matière grasse & 4,$34 ; 4,90 ; 5,42$ & 5,33 & 4,71 & 5,63 \\
\hline Protéines totales & 3,$4 ; 3,67 ; 4,06$ & 3,78 & 4,29 & 3,77 \\
\hline Caséine & 2,$30 ; 2,71 ; 3,02$ & & & 3,05 \\
\hline Protéines solubles & 0,$85 ; 0,92 ; 1,05$ & & & 0,71 \\
\hline Lactose & 3,$94 ; 4,31 ; 4,52$ & & & 4,76 \\
\hline Extrait sec & 13,$21 ; 13,65 ; 14,32$ & & & 14,8 \\
\hline MS dégraissée & 8,$88 ; 8,76 ; 8,90$ & 9,44 & 9,66 & 9,16 \\
\hline Cendre & 0,$80 ; 0,85 ; 0,89$ & & & 0,73 \\
\hline Calcium & 0,$112 ; 0,123 ; 0,137$ & & & \\
\hline Acidité & 0,$16 ; 0,17 ; 0,18$ & & & 0,17 \\
\hline $\mathrm{pH}$ & 6,$45 ; 6,54 ; 6,60$ & & & 6,52 \\
\hline Densité & 1,$0302 ; 1,0305 ; 1,039$ & & & 1,031 \\
\hline Point de congélation & & 0,574 & 0,565 & \\
\hline$N^{\circ}$ échantillons de l'usine & 30 & 263 & 226 & 19 \\
\hline
\end{tabular}

Tableau IV. Composition du lait de chèvre de la race Alpine (1), selon Anifantakis et Kandarakis (1980) et Voutsinas et al (1990) (2) et celle d'une race locale selon Simos et al (1991).

Composition of goat's milk from the Alpine breed (Anifantakis and Kandarakis, 1980 [1] ; Voutsinas et al, 1990 [2]) and from a local breed (Simos et al, 1991).

\begin{tabular}{|c|c|c|c|}
\hline & Alpine (1) & $\begin{array}{l}\text { Races } \\
\text { Alpine (2) }\end{array}$ & Locale (Metsovo) \\
\hline Matière grasse & 3,00 & $2,6-3,44-4,9$ & $4,45-5,18-5,8$ \\
\hline Protéines totales & 3,14 & $2,7-3,35-4,3$ & $3,46-3,56-3,69$ \\
\hline Caséine & 2,41 & $2,1-2,46-3,2$ & $2,72-2,80-2,88$ \\
\hline Protéines solubles & 0,73 & & \\
\hline Lactose & 4,33 & $3,9-4,3-4,7$ & $4,39-4,74-5,02$ \\
\hline Extrait sec & 11,13 & $10,6-11,76-13,5$ & $13,28-14,12-14,55$ \\
\hline MS dégraissée & 8,13 & $7,6-8,32-9,0$ & $8,51-8,94-9,20$ \\
\hline Cendre & 0,80 & $0,68-0,79-0,85$ & $0,69-0,76-0,82$ \\
\hline Calcium & & & 141 \\
\hline Acidité & 0,14 & $0,14-0,16-0,18$ & $0,14-0,19-0,17$ \\
\hline $\mathrm{pH}$ & 6,59 & $6,5-6,57-6,70$ & $6,32-6,55-6,68$ \\
\hline Densité & 1,030 & $1,027-1,030-1,032$ & $1,029-1,030-1,031$ \\
\hline$N^{\circ}$ échantillons & 32 & 35 & \\
\hline
\end{tabular}


approche de celle du lait de brebis, mais le rendement par lactation est faible : 70$120 \mathrm{~kg}$.

Un inconvénient majeur des races locales est leur durée limitée de lactation ( 5 à 6 mois) (fig 1).

La race Alpine s'adapte difficilement en Grèce, en raison de ses exigences alimentaires, mais le rendement par lactation atteint $200-400 \mathrm{~kg}$.

La composition du lait de la race Alpine est similaire à celle de celui produit par des animaux élevés dans les autres pays.

\section{Yoghourt et beurre}

Du yoghourt et du beurre sont préparés à une échelle artisanale à partir du lait de chèvre. Le beurre est préparé surtout à l'état fondu et n'est apprécié que par les consommateurs locaux.

Le groupe du professeur Kehagias de I'École de technologie alimentaire d'Athènes a étudié les qualités du yoghourt préparé à partir du lait de chèvre. Les résultats les plus importants de ces études sont les suivants : la fermeté du yoghourt

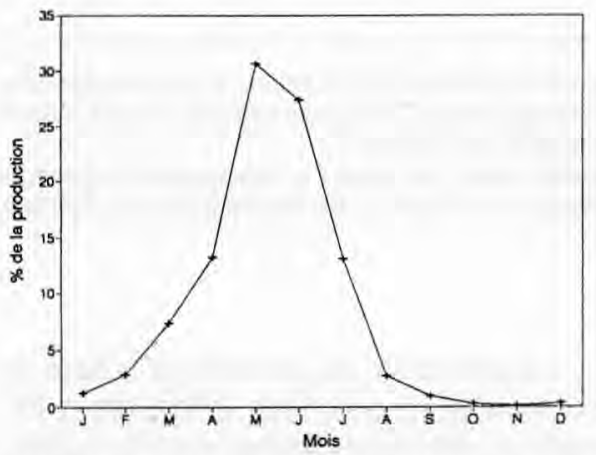

Fig 1. Distribution de la production du lait de chèvre pendant la lactation.

Distribution of goat's milk production during lactation. est identique à celle de celui obtenu à partir du lait de vache en raison de la richesse du taux protéique et butyreux du lait de chèvre, mais sa teneur en acétaldéhyde est inférieure ; l'homogénéisation du lait de chèvre améliore la qualité du yoghourt brassé; la comparaison des yoghourts égouttés à partir des 2 types de laits de vache et de chèvre fait apparaître une fermeté mesurée en force de pénétration plus élevée pour le produit de lait de vache $(82,67-43,67 ; \mathrm{pH}$ entre 4,3-4,1); mais le rendement est supérieur avec le lait de chèvre (Alexiou et al, 1988; Kehagias et al, 1981, 1987, 1989 a et b, 1992 a et b ; Giannoukou et al, 1989).

\section{Fromages}

L'addition du lait de chèvre au lait de brebis améliorerait les qualités organoleptiques du fromage Feta. Ce mélange n'est pas une falsification selon la législation grecque. Une étude récente de l'Institut du lait de lonannina (Mallatou et al, 1993) sur la préparation de fromage Feta à partir du lait pur chèvre et pur brebis, provenant de races locales et en mélange (tableau V) montre que le fromage fait uniquement avec du lait de chèvre avait la plus faible humidité, tandis que celui au lait de brebis en avait la plus haute.

Pour les fromages issus de lait de mélange, plus le pourcentage du lait de chèvre augmentait, plus l'humidité diminuait. Les $\mathrm{pH}$ étaient dans tous les cas identiques. Une autre différence significative était observée au niveau des rendements qui atteignaient 14,73 et $25,55 \mathrm{~kg}$ pour les laits pur chèvre et pur brebis respectivement (fromage de $60 \mathrm{j}$ ).

La teneur en acides gras libres des fromages de pur lait de chèvre était significativement la plus élevée. LitopoulouTzanetakis et Tzanetakis (1992) ont étudié la microflore du fromage Feta obtenu à par- 
Tableau V. Caractéristiques du fromage Feta obtenu à partir de différents types de lait selon Malatou et al.

Mean values of different variables for all stages (ages) studied of Feta cheeses made from different types of milk (Malatou et al).

\begin{tabular}{|c|c|c|c|c|c|c|}
\hline \multirow[t]{3}{*}{ Variable a } & \multicolumn{5}{|c|}{ Type de lait } & \multirow{3}{*}{$\begin{array}{l}\text { Erreur } \\
\text { standard b }\end{array}$} \\
\hline & \multicolumn{4}{|c|}{ Lait de chèvre } & \multirow{2}{*}{$\begin{array}{l}\text { Lait de brebis } \\
\qquad 100 \%\end{array}$} & \\
\hline & $100 \%$ & $75 \%$ & $50 \%$ & $25 \%$ & & \\
\hline Humidité (\%) & 54,94 & 56,97 & 57,16 & 57,41 & 57,41 & 0,76 \\
\hline HFD (\%) & 72,22 & 73,24 & 73,54 & 72,24 & 72,71 & 1,10 \\
\hline Sel $(\%)$ & 2,72 & 2,66 & 2,69 & 2,82 & 2,84 & 0,12 \\
\hline Sel dans la phase aqueuse (\%) & 5,04 & 4,76 & 4,76 & 4,99 & 4,93 & 0,21 \\
\hline $\mathrm{pH}$ & 4,62 & 4,54 & 4,58 & 4,68 & 4,57 & 0,07 \\
\hline Acidité (\%) & 1,06 & 1,04 & 1,14 & 1,19 & 1,11 & 0,19 \\
\hline Protéines (\%) & 16,79 & 16,52 & 16,59 & 16,06 & 17,03 & 0,50 \\
\hline Matière grasse $(\%)$ & 24,42 & 22,22 & 22,29 & 21,15 & 21,05 & 0,66 \\
\hline Gras/sec (\%) & 54,28 & 51,63 & 52,04 & 49,68 & 48,95 & 1,59 \\
\hline Rendement & 15,07 & 17,78 & 21,14 & 24,56 & 27,93 & 0,62 \\
\hline Rendement à $56 \%$ d'humidité & 15,34 & 17,58 & 20,53 & 23,70 & 26,91 & 0,71 \\
\hline NT/MS (\%) & 5,84 & 6,02 & 6,07 & 5,89 & 6,28 & 0,20 \\
\hline Ns eau/NT (\%) & 15,61 & 15,46 & 17,25 & 16,35 & 16,73 & 0,58 \\
\hline Ns TCANTT (\%) & 11,08 & 10,87 & 11,92 & 12,90 & 11,51 & 0,90 \\
\hline Ns PTA/NT (\%) & 3,05 & 2,56 & 2,90 & 2,71 & 2,03 & 0,17 \\
\hline Acidité & 2,25 & 2,23 & 1,78 & 1,58 & 1,46 & 0,14 \\
\hline Apparence & 8,50 & 8,42 & 8,07 & 8,10 & 8,87 & 0,46 \\
\hline Texture & 33,80 & 31,60 & 31,30 & 31,70 & 36,10 & 1,84 \\
\hline Flaveur & 41,50 & 38,80 & 36,12 & 37,50 & 43,75 & 3,04 \\
\hline $\begin{array}{l}\text { Caractéristiques organoleptiques } \\
\text { totales }\end{array}$ & 83,80 & 78,82 & 75,49 & 77,30 & 88,72 & 5,07 \\
\hline Fermeté (organoleptique) & 5,18 & 4,43 & 4,24 & 4,05 & 3,87 & 0,28 \\
\hline Force au point de fracture & 1,10 & 0,89 & 0,77 & 0,71 & 1,47 & 0,27 \\
\hline $\begin{array}{l}\text { \% de compression au point } \\
\text { de fracture }\end{array}$ & 21,50 & 20,75 & 20,23 & 19,16 & 18,13 & 1,09 \\
\hline Fermeté (rhéologie) & 2,59 & 2,23 & 1,18 & 1,81 & 2,22 & 0,19 \\
\hline
\end{tabular}

a $\mathrm{HFD}=$ humidité dans le fromage dégraissé $; \mathrm{NT} / \mathrm{MS}=$ azote total/matière sèche $; \mathrm{Ns}$ eau/NT = azote soluble dans l'eau/azote total ; Ns TCA/NT = azote soluble dans l'acide trichloroacétique à $12 \%$ azote total; Ns PTA/NT = azote soluble dans l'acide phosphotungstique à $5 \%$ azote total ; ${ }^{\mathrm{b}}$ d'après la table ANOVA.

a $H F D=$ moisture in defatted cheese $; N T / M S=$ total nitrogen/dry matter $;$ Ns eau/NT = watersoluble nitrogen/total nitrogen ; Ns TCA/NT = soluble nitrogen in 12\% TCA/total nitrogen ; Ns PTA/NT = soluble nitrogen in 5\% PTA/total nitrogen; ${ }^{\circ}$ from the ANOVA table.

tir du lait cru de chèvre, pendant l'affinage. lls ont montré que pendant les premiers jours, la flore prédominante était constituée de bactéries lactiques mais à $90 \mathrm{j}, L$ plantarum, $E$ faecalis et durans, et $L$ paracasei deviennent prédominants.
Le laboratoire de technologie laitière de I'Université agronomique d'Athènes a développé des technologies pour la fabrication de nouveaux fromages:

- le fromage Fildrus, version du fromage Kefalotyri, obtenu à partir du lait de 
chèvre: la technologie de ce fromage est présentée au tableau $\mathrm{VI}$; ce fromage a fait l'objet d'un brevet gréco-bulgare (Baltadjieva et al, 1978) ;

- le fromage Rumelia à pâte filée de type Kachkaval fait à partir de lait de chèvre a lui aussi fait l'objet d'un brevet grécobulgare (Baltadjieva et al, 1980, 1984) ; les données analytiques de ce fromage sont présentées au tableau VII ;

- un fromage à pâte demi-dure, type Edam, est produit en petites quantités à

Tableau VI. Diagramme de fabrication du fromage Fildrus.

Fildrus cheese-making diagram.

Étapes de fabrication

Traitement initial du lait

Pasteurisation $75-76^{\circ} \mathrm{C} 20-30 \mathrm{~min}$

Standardisation $38 \mathrm{~g} / \mathrm{l}$

Addition de levains

Lb bulgaricus : Str thermophilus 1:2

$0,3-0,5 \%$

$34-35^{\circ} \mathrm{C}$

Coagulation

35-40 min

$34^{\circ}-35^{\circ} \mathrm{C}$

Décaillage

Grains du ble

\section{Réchauffage}

Soutirage de $2 / 3$ du lactosérum

Addition de l'eau salée : $10 \%$ quantité de lait

Température finale : $46^{\circ}-48^{\circ} \mathrm{C}$

Brassage après le feu

$46^{\circ}-48^{\circ} \mathrm{C}$

$15-20 \mathrm{~min}$

\section{Pressage}

$3 \mathrm{~h}-20-22^{\circ} \mathrm{C}$

$20-25 \mathrm{~kg} / 1 \mathrm{~kg}$ de fromage

\section{Salage}

$\mathrm{Sec}$

2 fois tous les 3 j

\section{Affinage}

Cave tempérée : $14^{\circ} \mathrm{C}, 50 \mathrm{j}$

Humidité : 85-90\%

\section{Conditionnement}

Après $50 \mathrm{j}$ paraffinage

et enrobage avec film de polyéthylène

\section{Contrôles}

Acidité, $\mathrm{pH}$

18-19D

$20: 22^{\circ} \mathrm{D}$

Acidité, $\mathrm{pH}$
Humidité $40 \%$

Mat Gras/Ex sec :

$52,5 \%$

$\mathrm{NaCl}: 2,8 \%$ 
Tableau VII. Acides aminés libres du fromage Rumelia ( $\mathrm{mg} / 100 \mathrm{~g}$ de fromage).

Free amino acids in Rumelia cheese ( $\mathrm{mg} / 100 \mathrm{~g}$ cheese).

\begin{tabular}{|c|c|c|c|c|c|c|c|c|}
\hline Acides aminés & 1 mois & $\%$ du total & 2 mois & $\%$ du total & 3 mois & $\%$ du total & \multicolumn{2}{|c|}{$\%$ du total } \\
\hline Lysine & $68,49 \pm 4,30$ & 11,60 & $161,96 \pm 16,77$ & 14,20 & $383,85 \pm 23,92$ & 12,13 & $39,21 \pm 19,80$ & 11,60 \\
\hline Histidine & $14,79 \pm 2,58$ & 2,50 & $28,52 \pm 3,78$ & 2,51 & $82,40 \pm 5,17$ & 2,60 & $86,35 \pm 5,20$ & 2,60 \\
\hline Arginine & $1,61 \pm 0,49$ & 0,27 & $5,95 \pm 1,71$ & 0,52 & $23,63 \pm 3,16$ & 0,75 & $25,44 \pm 2,14$ & 0,76 \\
\hline Acide aspartique & $10,05 \pm 1,23$ & 1,70 & $20,51 \pm 1,88$ & 1,30 & $52,73 \pm 3,95$ & 1,67 & $53,08 \pm 2,34$ & 1,58 \\
\hline Thréonine & $63,40 \pm 6,31$ & 10,77 & $119,11 \pm 7,84$ & 10,47 & $159,83 \pm 9,15$ & 5,05 & $164,24 \pm 8,22$ & 4,89 \\
\hline Sérine & $49,05 \pm 4,64$ & 8,30 & $91,20 \pm 6,12$ & 8,00 & $215,02 \pm 14,45$ & 6,80 & $214,63 \pm 9,08$ & 6,40 \\
\hline Acide glutamique & $91,34 \pm 9,76$ & 15,50 & $159,84 \pm 22,57$ & 14,05 & $655,01 \pm 49,54$ & 20,70 & $713,22 \pm 33,97$ & 21,23 \\
\hline Proline & $50,50 \pm 5,68$ & 8,57 & $122,15 \pm 11,44$ & 10,74 & $176,03 \pm 6,61$ & 5,56 & $189,78 \pm 6,73$ & 5,65 \\
\hline Glycine & $29,17 \pm 3,81$ & 4,95 & $35,59 \pm 3,98$ & 3,13 & $110,69 \pm 10,48$ & 3,50 & $127,78 \pm 15,93$ & 3,80 \\
\hline Alanine & $14,02 \pm 1,44$ & 2,38 & $30,99 \pm 1,50$ & 2,72 & $105,47 \pm 5,61$ & 3,30 & $122,19 \pm 4,27$ & 3,64 \\
\hline Valine & $39,90 \pm 3,60$ & 6,77 & $75,40 \pm 5,58$ & 6,63 & $263,31 \pm 13,80$ & 8,32 & $307,42 \pm 27,18$ & 9,15 \\
\hline Methionine & $14,37 \pm 1,45$ & 2,44 & $21,51 \pm 2,51$ & 1,90 & $111,94 \pm 8,21$ & 3,54 & $116,70 \pm 5,26$ & 3,47 \\
\hline Isoleucine & $27,15 \pm 2,59$ & 4,61 & $55,69 \pm 3,04$ & 4,90 & $203,22 \pm 10,11$ & 6,42 & $207,70 \pm 7,37$ & 6,18 \\
\hline Leucine & $69,23 \pm 3,27$ & 11,75 & $108,99 \pm 4,58$ & 9,50 & $389,14 \pm 15,40$ & 12,29 & $401,47 \pm 2,15$ & 11,95 \\
\hline Tyrosine & $12,90 \pm 1,38$ & 2,19 & $28,66 \pm 1,99$ & 2,50 & $49,51 \pm 2,30$ & 1,56 & $52,10 \pm 14,99$ & 1,55 \\
\hline Phenylalanine & $33,73 \pm 1,72$ & 5,73 & $71,49 \pm 6,20$ & 6,28 & $183,80 \pm 6,28$ & 5,81 & 185,06 & 5,51 \\
\hline Total & 589,68 & $\approx 100$ & 1137,55 & $\approx 100$ & 3165,56 & $\approx 100$ & 3359,37 & $\approx 100$ \\
\hline
\end{tabular}


I'usine Dodon ; les tableaux VIII et IX présentent la composition en acides aminés et en acides gras libres de ce fromage pendant l'affinage (Kalantzopoulos et al, 1983 ; Baltadjieva et al, 1985).

Le lait de chèvre est également utilisé pour l'amélioration de la texture et de la qualité des fromages de lactosérum. Pendant le chauffage, 5 à $25 \%$ de lait de chèvre sont ajoutés avant la coagulation complète des protéines solubles du lactosérum. Cette variété de fromage est très appréciée des consommateurs grecs.

\section{CONCLUSION}

Les régions dans lesquelles se fait l'élevage de chèvre en Grèce sont généralement défavorisées, caractérisées par une répartition très disséminée des exploitations agricoles et par des troupeaux souvent mixtes chèvre-brebis. $\mathrm{Ce}$ sont des lieux de grande tradition pastorale, où il devient nécessaire d'améliorer le cheptel caprin pour obtenir une meilleure rentabilité économique.

Les recherches en cours concernent l'étude des populations et races indigènes (amélioration génétique, alimentation, méthode de conduite d'élevage, etc...).

Elles concernent aussi des recherches sur l'amélioration de la qualité du lait et l'amélioration des fabrications fromagères traditionnelles et une meilleure valorisation du lait.

Les efforts accomplis ont conduit à une augmentation significative du cheptel caprin et de la production du lait. Le grand intérêt des consommateurs pour le fromage

Tableau VIII. Acides gras libres volatils du fromage type Edam.

Volatile free fatty acids in Edam-type cheese.

\begin{tabular}{|c|c|c|c|c|c|c|c|c|c|}
\hline \multirow[t]{2}{*}{ Acides gras } & & \multicolumn{2}{|c|}{1 mois } & \multicolumn{2}{|c|}{2 mois } & \multicolumn{2}{|c|}{3 mois } & \multicolumn{2}{|c|}{4 mois } \\
\hline & & $g / k g$ & $\begin{array}{l}\% d u \\
\text { total }\end{array}$ & $g / \mathrm{kg}$ & $\begin{array}{l}\% d u \\
\text { total }\end{array}$ & $g / k g$ & $\begin{array}{l}\% d u \\
\text { total }\end{array}$ & $g / \mathrm{kg}$ & $\begin{array}{l}\% d u \\
\text { total }\end{array}$ \\
\hline Acétique & $\begin{array}{l}\text { Moyenne } \\
\text { Min-Max }\end{array}$ & $\begin{array}{c}0,176 \\
0,15-0,19\end{array}$ & 7,2 & $\begin{array}{c}0,345 \\
0,32-0,37\end{array}$ & 10,5 & $\begin{array}{c}0,257 \\
0,23-0,28\end{array}$ & 6,5 & $\begin{array}{c}0,219 \\
0,20-0,23\end{array}$ & 4,8 \\
\hline Propionique & $\begin{array}{l}\text { Moyenne } \\
\text { Min-Max }\end{array}$ & $\begin{array}{c}0,122 \\
0,10-0,13\end{array}$ & 5,0 & $\begin{array}{c}0,189 \\
0,16-0,22\end{array}$ & 5,7 & $\begin{array}{c}0,154 \\
-\end{array}$ & 3,9 & $\begin{array}{c}0,105 \\
0,09-0,12\end{array}$ & 2,3 \\
\hline Isobutyrique & $\begin{array}{l}\text { Moyenne } \\
\text { Min-Max }\end{array}$ & $\begin{array}{c}0,215 \\
0,20-0,23\end{array}$ & 8,8 & $\begin{array}{c}0,252 \\
0,22-0,28\end{array}$ & 7,6 & $\begin{array}{c}0,182 \\
0,17-0,20\end{array}$ & 4,6 & $\begin{array}{c}0,128 \\
0,11-0,31\end{array}$ & 2,8 \\
\hline Butyrique & $\begin{array}{l}\text { Moyenne } \\
\text { Min-Max }\end{array}$ & $\begin{array}{c}0,527 \\
0,48-0,54\end{array}$ & 21,6 & $\begin{array}{c}0,789 \\
0,76-0,85\end{array}$ & 23,8 & $\begin{array}{c}1,046 \\
0,91-1,12\end{array}$ & 26,5 & $\begin{array}{c}1,252 \\
1,13-1,31\end{array}$ & 27,4 \\
\hline Isovalérique & $\begin{array}{l}\text { Moyenne } \\
\text { Min-Max }\end{array}$ & $\begin{array}{c}0,383 \\
0,35-0,41\end{array}$ & 15,7 & $\begin{array}{c}0,285 \\
0,26-0,30\end{array}$ & 8,6 & $\begin{array}{c}0,300 \\
0,28-0,31\end{array}$ & 7,6 & $\begin{array}{c}0,187 \\
0,17-0,19\end{array}$ & 4,1 \\
\hline Valérique & $\begin{array}{l}\text { Moyenne } \\
\text { Min-Max }\end{array}$ & $\begin{array}{c}0,078 \\
0,06-0,09\end{array}$ & 3,2 & $\begin{array}{c}0,089 \\
0,08-0,09\end{array}$ & 2,7 & $\begin{array}{c}0,075 \\
0,07-0,08\end{array}$ & 1,9 & $\begin{array}{c}0,037 \\
0,03-0,04\end{array}$ & 0,8 \\
\hline Caproïque & $\begin{array}{l}\text { Moyenne } \\
\text { Min-Max }\end{array}$ & $\begin{array}{c}0,536 \\
0,45-0,60\end{array}$ & 22,0 & $\begin{array}{c}0,772 \\
0,75-0,80\end{array}$ & 23,3 & $\begin{array}{c}1,113 \\
1,01-1,24\end{array}$ & 28,2 & $\begin{array}{c}1,489 \\
1,28-1,56\end{array}$ & 32,6 \\
\hline Caprylique & $\begin{array}{l}\text { Moyenne } \\
\text { Min-Max }\end{array}$ & $\begin{array}{c}0,402 \\
0,38-0,43\end{array}$ & 16,5 & $\begin{array}{c}0,593 \\
0,54-0,63\end{array}$ & 17,9 & $\begin{array}{c}0,821 \\
0,74-0,89\end{array}$ & 20,8 & $\begin{array}{c}1,151 \\
1,06-1,29\end{array}$ & 25,2 \\
\hline Total & $\begin{array}{l}\text { Moyenne } \\
\text { Min-Max }\end{array}$ & $\begin{array}{c}2,439 \\
2,26-2,67\end{array}$ & 100 & $\begin{array}{c}3,315 \\
3,12-3,58\end{array}$ & $\approx 100$ & $\begin{array}{c}3,949 \\
3,75-4,12\end{array}$ & 100 & $\begin{array}{c}4,569 \\
4,36-4,79\end{array}$ & 100 \\
\hline
\end{tabular}


Tableau IX. Acides aminés libres du fromage type Edam ( $\mathrm{mg} / 100 \mathrm{~g}$ de fromage). Free amino acids in Edam-type cheese ( $\mathrm{mg} / 100 \mathrm{~g}$ cheese).

\begin{tabular}{|c|c|c|c|c|c|c|c|c|}
\hline Acides aminés & 1 mois & $\%$ du total & 2 mois & $\%$ du total & 3 mois & $\%$ du total & 4 mois & $\%$ du total \\
\hline Lysine & $51,00 \pm 2,98$ & 10,49 & $75,95 \pm 3,15$ & 8,06 & $266,98 \pm 12,16$ & 10,91 & $410,34 \pm 15,51$ & 14,03 \\
\hline Histidine & $16,42 \pm 1,31$ & 3,38 & $34,27 \pm 1,60$ & 3,64 & $103,19 \pm 3,52$ & 4,22 & $120,25 \pm 5,52$ & 4,11 \\
\hline Arginine & $1,26 \pm 0,20$ & 0,26 & $5,59 \pm 0,99$ & 0,59 & $11,28 \pm 1,13$ & 0,46 & $21,65 \pm 0,65$ & 0,74 \\
\hline Acide aspartique & $9,94 \pm 1,05$ & 2,04 & $46,37 \pm 1,98$ & 4,92 & $89,81 \pm 3,66$ & 3,67 & $121,73 \pm 6,23$ & 4,16 \\
\hline Thréonine & $39,27 \pm 1,99$ & 8,08 & $50,15 \pm 2,29$ & 5,33 & $117,46 \pm 6,20$ & 4,80 & $140,38 \pm 5,61$ & 4,80 \\
\hline Sérine & $29,32 \pm 1,44$ & 6,03 & $71,25 \pm 3,01$ & 7,57 & $97,73 \pm 8,91$ & 3,87 & $106,15 \pm 6,63$ & 3,63 \\
\hline Acide glutamique & $67,99 \pm 2,30$ & 13,98 & $142,63 \pm 7,41$ & 15,15 & $520,16 \pm 25,53$ & 21,25 & $591,59 \pm 9,51$ & 20,23 \\
\hline Proline & $38,92 \pm 1,18$ & 8,00 & $77,76 \pm 2,64$ & 8,26 & $130,80 \pm 5,89$ & 5,34 & $171,90 \pm 9,65$ & 5,88 \\
\hline Glycine & $15,59 \pm 0,85$ & 3,21 & $41,10 \pm 2,78$ & 4,36 & $108,60 \pm 4,00$ & 4,44 & $124,47 \pm 5,08$ & 4,26 \\
\hline Alanine & $15,55 \pm 0,72$ & 3,20 & $51,37 \pm 3,04$ & 5,45 & $81,11 \pm 3,10$ & 3,31 & $97,01 \pm 4,80$ & 3,32 \\
\hline Valine & $44,56 \pm 1,85$ & 9,16 & $81,96 \pm 2,59$ & 8,70 & $239,93 \pm 12,88$ & 9,80 & $265,43 \pm 16,74$ & 9,07 \\
\hline Methionine & $13,89 \pm 0,85$ & 2,86 & $17,86 \pm 2,00$ & 1,90 & $86,10 \pm 5,72$ & 3,52 & $93,68 \pm 3,69$ & 3,20 \\
\hline Isoleucine & $21,86 \pm 1,28$ & 4,50 & $63,07 \pm 3,32$ & 6,70 & $130,76 \pm 7,14$ & 5,34 & $152,42 \pm 12,58$ & 5,21 \\
\hline Leucine & $84,86 \pm 4,15$ & 17,45 & $113,43 \pm 6,66$ & 12,04 & $295,58 \pm 14,31$ & 12,07 & $328,12 \pm 14,10$ & 11,22 \\
\hline Tyrosine & $2,75 \pm 0,86$ & 0,57 & $10,30 \pm 1,30$ & 1,09 & $30,76 \pm 1,90$ & 1,26 & $38,55 \pm 2,79$ & 1,32 \\
\hline Phenylalanine & $33,58 \pm 1,31$ & 6,91 & $58,69 \pm 1,84$ & 6,23 & $132,54 \pm 5,76$ & 5,41 & $147,57 \pm 6,45$ & 5,05 \\
\hline Total & 486,21 & $\approx 100$ & 941,76 & $\approx 100$ & 2448,04 & $\approx 100$ & 2924,96 & $\approx 100$ \\
\hline
\end{tabular}


Feta, ainsi que le prix élevé de ce fromage, ne permettent pas d'envisager la production de fromages pur-chèvre, sauf à des fins artisanales ou familiales.

\section{RÉFÉRENCES}

Alexiou C, Kehagias C, Konidari P, Lambroukos M, Garrifalidis N (1988) Rheological properties of stirred yoghurt from goats milk. Greek J Dairy Sci Technol 1, 20-29

Anifantakis E, Kandarakis J (1980) Contribution to the study of the composition of goats milk. Milchwissenschaft 35, 617-619

Anifantakis E, Giannakopoulou K, Vastardis J, Gardika A, Fiskatoris M (1993) The quality of raw goats milk of the regions of Epirus and Pelopenesus of Greece. Greek J Dairy Sci Technol (in press)

Baltadjieva M, Stamenova V, Veinoglou B, Anifantakis $E$ (1978) Patent for the production of Fildrus cheese from goats milk $n^{\circ} 40880$. Institute for Inventions and Innovations, Sofia Bulgaria

Baltadjieva M, Stamenova V, Veinoglou B, Kalantzopoulos G (1980) Application for an invention of the production of Rumelia cheese from goats milk $n^{\circ} 3163$. Institute for Inventions and Innovations, Sofia Bulgaria

Baltadjieva M, Veinoglou B, Kalantzopoulos G, Stamenova V, Sfakianos A (1984) Un nuovo tipo di fromaggio "Rumelia", di latte di capra. Sci Tec Latt-Casearia 35, 451-464

Baltadjieva M, Kalantzopoulous G, Stamenova V, Sfakianos A (1985) Composition en acides gras libres et en acides aminés de 2 fromages fabriqués à partir de lait de chèvre. Lait 65, 221-241

Giannoukou M, Kehagias C, Katsipis A (1989) Effect of various factors on the characteristics and the yield of cheese coagulum and strained yoghurt from various kinds of milk. Greek J Dairy Sci Technol 4, 527-535

Kalantzopoulos G, Veinoglou B, Baltadjieva M, Alexandropoulos C, Stamenova V, Sfakianos A (1983) Préparation d'un fromage semi-dur à partir du lait de chèvre. Lait $63,354-361$
Kehagias C, Dalles T (1981) Functional properties of sodium caseinates derived from ewes, goats and cows milk. Milchwissenschaft 36 , 29-31

Kehagias C, Konidari P, Laskaris C, Kazazis J (1987) Fermentation of cows, goats and ewes milk by thermophilic acid producing cuttures. Greek J Dairy Sci Technol 1, 43-59

Kehagias C, Zervoudakis S, Koulouri S, Kazazis $J$ (1989a) Effect of various technological parameters on the characteristics of yoghurt from goats milk. Greek J Dairy Sci Technol 3, 395-403

Kehagias C, Zervoudaki A, Parlama C (1989b) Influence of the composition and additives on properties of set-type yoghurt from goat milk. Small Ruminant Res 2, 35-45

Kehagias C, Kalavritinos L, Triadopoulou C (1992a) Effect of $\mathrm{pH}$ on the yield and solids recovery of strained yoghurt from goat and cow milk. Cult Dairy Prod J 27, 10, 12-14

Kehagias C, Agelidou M, Kremastos M, Koulouiris S (1992b) Characteristics of Feta and Teleme cheese curds from goats and cow milk fermented with various starters. V. Int Conf Goats India, March 387

Litopoulou-Tzanetakis E, Tzanetakis N (1992) Microbiological study of white-brined cheese made from raw goat milk. Food Microbiol 9, 13-19

Mallatou E, Pappas C, Voutsinas L (1993) Manufacture of Feta cheese from sheeps milk, goats milk and different mixtures. Int Dairy $J$ (in press)

Service national statistique de la Grèce (1991) Le cheptel animal, bovin, ovin, caprin, 43-54

Simos E, Voutsinas L, Pappas C (1991) Composition of milk of native Greek goats in the region of Metsovo. Small Ruminant Res 4, 4760

Veinoglou B, Baltadjieva M, Kalantzopoulos G, Stamenova V, Papadopoulou E (1982) La composition du lait de chèvre de la région de Plovdiv en Bulgarie et de loannina en Grèce. Lait 62, 155-165

Voutsinas L, Pappas C, Katsiari M (1990) The composition of Alpine goats milk during lactation in Greece. J Dairy Res 57, 41-51 